\title{
History of the Emergence and Spatiotemporal Spread of Tobacco Mosaic Virus in China
}

\section{Madzokere ET}

Institute for Glycomics, Griffith University, Gold Coast Campus, Southport, QLD 4215, Australia

*Corresponding author: Madzokere ET, Institute for Glycomics, Griffith University, Gold Coast Campus, Southport, QLD 4215 , Australia; Tel: +263 78 517 8490; E-mail: Madzokt@yahoo.com

Received date: October 23, 2017; Accepted date: November 23, 2017; Published date: December 04, 2017

Copyright: () 2017 Madzokere ET. This is an open-access article distributed under the terms of the Creative Commons Attribution License, which permits unrestricted use, distribution, and reproduction in any medium, provided the original author and source are credited.

\begin{abstract}
The ongoing spread of Tobacco mosaic virus (TMV) throughout China threatens and diminishes proceeds from production of tobacco and other crops. Determining how and when TMV first emerged in China, its current evolutionary rate, diffusion pathways, spatial and plant host distributions, can help minimize the risk associated with mosaic disease (MD). Here, 110 TMV Coat Protein (CP) gene sequences sampled from 12 distinct plant hosts between 1997 and 2015 from 18 geographical locations within China (14 Provinces, two Municipalities and two Autonomous regions) were used in a probabilistic Bayesian inferential framework implemented in BEAST v1.8.1 to reconstruct TMV's evolutionary history from emergence to spatiotemporal diffusion. This entailed estimating and inferring; (a) the time when and location where TMV's most recent common ancestor (MRCA) emerged, (b) the evolutionary rate, (c) diffusion pathways, (d) levels of genetic diversity and, (e) phylogenetic relationships amongst viruses. This study infers that TMV emerged around 1924 (95\% HPD; 1860 to 1971) in Henan province. Its mean nucleotide substitution rate of $1.09 \times 10^{-3}$ is marginally higher than previous TMV and Tobamovirus species rates. TMV's current wide spatial and plant host distribution across China is due largely to (i) utilization of 15 Bayes factor supported diffusion pathways, $60 \%$ of which were outward bound viral movements from Yunnan province to proximal and distant sampling locations and (ii) a growing shift toward cost-efficient tobacco crop substitution alternatives and adoption of a mixed-crop farming system. These analyses also suggest that Yunnan province is most probably both a source rather than a sink of TMV dispersal throughout China and a major thoroughfare of trans-China TMV movements. Finally, results also indicate that TMV populations exhibited both low effective population sizes and levels of genetic diversity, while individuals from distinct hosts were phylogenetically similar probably due to strong bottlenecks and purifying selection.
\end{abstract}

Keywords: Tobacco mosaic virus; Bayesian; Phylogeographic diffusion; Genetic diversity

\section{Introduction}

China is the world's largest producer and consumer of tobacco (Nicotiana tabacum L. sp), an important economic crop in China and worldwide [1]. Tobacco farming is very labor intensive. Its cultivation in China begins in summer and harvesting occurs in mid-August. Although most tobacco varieties grown in China are traded for prices lower than those for cotton, rice or sugarcane [2], the tobacco industry in China remains one of the most important sources of tax revenue for central government [3]. Principally, Chinese farmers grow tobacco widely because; (i) it has a far more limited market and price risk as compared to other agricultural crops since it is sold on contract at a guaranteed price and, (ii) cash payouts by the government are usually prompt and uncomplicated upon delivery of tobacco [2]. The 2016 release of the International Committee on taxonomy of Viruses (ICTV; http://www.ictvonline.org/virustaxonomy.asp), classifies Tobacco mosaic virus (TMV), the causal agent of mosaic disease (MD), within the genus Tobamovirus under the Virgaviridae family. This genus currently holds 37 viral species (ICTV; http://www.ictvonline.org/ virustaxonomy.asp). Infection of tobacco and other crops by TMV, greatly diminishes crop yields, financial returns, and threatens farmer livelihoods throughout China [4].
Tobacco mosaic virus (TMV) infects more than 350 plant species within nine plant families of nutritional, economic, horticultural and ornamental importance [4-6]. Here, weeds and members of the Solanaceae (e.g. tobacco, tomato, potato, eggplant, pepper, and petunia), Brassicaceae (e.g. cabbage, radish), Cucurbitaceae (e.g. cucumber, watermelon, zuchini, pumpkin), and Legumineae (e.g. bean) plant families, as well as ornamentals such as chrysanthemums (Chrysanthemum indicum L.), and impatiens (Impatiens balsamina) [7-11], are most susceptible to TMV. The virus is rarely seed-borne and is not vector-transmitted $[12,13]$. In addition to transmission through susceptible weeds and plant reservoirs, TMV can also be transmitted mechanically through use of contaminated cutting tools, handling, plant cuttings, grafting, contact between plants or other contaminated surfaces, soil and/or potting material [5]. Surveys conducted in China and Turkey have shown that, the risk of MD outbreaks occurring elevates with an increase in TMV reservoirs $[1,8]$. Mosaic disease (MD) symptoms include, leaf necrosis, curling, malformations, mosaic patterns, stunted growth, and loss of apical dominance which leads to poor flower and fruit development [4,5]. The spread of TMV may be reduced through increased use of pathogen free plantlets or cuttings, biological pesticides, scouting, rouging, disinfecting tools, benches and surfaces from the greenhouse to the field $[5,14]$.

Tobacco was first introduced to China from the West in the $16^{\text {th }}$ and $17^{\text {th }}$ centuries via three routes; (i) the Philippines and Vietnam to the south of China, (ii) South Korea to the northeast of China, and (iii) Japan to the east of China [15]. Also, in May of 1858, China's Qing 
Dynasty's Xianfeng Emperor authorized negotiations with delegations representing interests of Russia, the Second French Empires, the United Kingdom and the United States culminating in the signing of a collective of documents in June 1858, described as the Treaty of Tianjin. Reluctant ratification of this seemingly unequal Treaty by the Emperor of China in the Convention of Peking in 1860 opened Chinese ports to foreign trade, legalized the import of opium and paved the way for duty-free imports of tobacco seed and cigarettes into China $[16,17]$. By 1890 , the first machine-made cigarettes had reached China courtesy of the American Tobacco Company, later known as British American Tobacco Company (BAT) [18].

From its founding in 1902, BAT maintained a lucrative monopoly of the market until 1915 when Nanyang Brothers Tobacco Company (NBTC), whose headquarters were in Hong Kong and Shanghai, entered China and extended its operations into the country's major markets despite the use of aggressive tactics against it [19]. Both companies grew rapidly during the 1920s, and competition between them reached its peak, but by 1930 NBTC weakened, bringing an end to serious commercial rivalry. Foreign tobacco companies such as BAT in China were financially fruitful at this time because, they maximized tobacco cigarette sales and brought-in agricultural specialists to breed tobacco for improved production. By 1934, an estimated 300000 rural Chinese households were cultivating tobacco to sell to BAT [18]. While public records suggest that tobacco cultivation was firmly established by the mid-1800s in China [20], no exact information is given on where and/or when, the first tobacco crop was cultivated. It is widely known though that China's modern production of tobacco for cigarettes began in 1914 when BAT introduced tobacco seed to Yunnan province and planted in Yuxi County the following year [21]. Although the Sino-Japanese War (1937-1945) disrupted cigarette supplies to China, their popularity had taken hold [17]. When the People's Republic of China (PRC) was founded in 1949, the tobacco industry was nationalized and strong relationships between the central government and cigarette manufacturers in the provinces were formed $[17,22]$.

Soon after 1950, most foreign owned tobacco companies including BAT were forced out of China by the PRC because of suspected meddling in and influencing of China's socio-political and economic affairs; for purportedly participating in illicit dealings with the Kuomintang and the Chinese Communist Party, and for instigating diplomatic incidents, boycotts, strikes, student protests, and exploiting relations with peasant tobacco growers [19]. With limited provincial and regional control of tobacco and cigarette production in China, profits from the tobacco industry between 1950 and 1977 remained flat [18]. To reassert tobacco control within her borders, China founded the China National Tobacco Corporation (CNTC) in 1981 [23], and shortly thereafter in 1983, it also established the State Tobacco Monopoly Administration (STMA) [24]. The CNTC is the umbrella company for approximately 31 smaller tobacco companies in China, and it is responsible for centralized management of staff, finance, properties, products, supply, distribution, and domestic and foreign trade of the country's tobacco industry [20,25]. The STMA in conjunction with CNTC, determines the overall government tobacco policy, including tobacco leaf quotas, pricing, cigarette production quotas, and it also controls tobacco production, marketing, imports, and exports [26,27].

To this day, the CNTC is China's and the world's largest manufacturer of tobacco products. It receives jurisdiction from the STMA to enforce tobacco monopoly in China on marketing, production, distribution, and sales of tobacco products [27]. During the Deng Xiaoping era (1978-1997), China's tobacco and cigarette production industry experienced rapid growth as manufacturers actively competed with one another for foreign currency to purchase cutting edge European equipment and newer varieties of tobacco seed stock [22]. Currently, all the four largest transnational tobacco companies; Philip Morris International (PMI), British American Tobacco (BAT), Japan Tobacco International (JTI) and Imperial Tobacco (IT), have direct partnerships with the CNTC $[20,25,28]$.

Although twenty-seven of China's thirty-one tobacco provincial, municipal and autonomous region companies plant tobacco, tobacco production is largely concentrated in Yunnan, Guizhou, Henan, and Sichuan provinces [3]. Currently, these four tobacco production hubprovinces collectively account for over $60 \%$ of China's tobacco planting area $[1,3]$. Nevertheless, the majority of China's state administered tobacco industrial companies are distributed mostly within Yunnan, Hunan, Shanghai, Henan, Jiangsu, Zhejiang, Shandong and Guizhou provinces [29]. These tobacco companies have since been designated into three geographical groups; (i) the Shanghai group including Shanghai, Jiangsu, Zhejiang, Beijing, and Tianjin, (ii) the Yunnan group, including the northeastern and southwestern parts of China as well as Hainan and, (iii) the Hunan group, including Hunan, Hubei and central China $[3,20]$.

To minimize the financial burden incurred by farmers, in 2006, China's central government eliminated all agricultural taxes except for a special tobacco leaf tax, which was intentionally retained and redesigned [30]. China's central government declares that the special tobacco leaf tax is a macroeconomic instrument to control tobacco production and consumption and it is also a source of revenue for local government through the CNTC [31]. This decision angered tobacco farmers, most of whom continue to argue that the special tax further erodes their already meager proceeds. As a retaliatory response to this action by central government, throughout China, there has been an ongoing increase in substitution of tobacco for other crops and a shift toward mixed-cropping by traditional tobacco farmers [1]. As an example, in a 3-year pilot study that began in 2008 in Yunnan province, more than 450 traditionally tobacco farming families substituted the crop for others such as tomatoes, flowers, vegetables, arrowhead, and grapes and reported earning between $21 \%$ and $110 \%$ more per acre than they had been earning from tobacco farming alone [32]. Promotion of tobacco substitution and mixed-cropping on otherwise traditionally tobacco farms, fields and/or plantations increases the risk of sporadic TMV spread across China. So far, sixteen Chinese tobacco growing provinces have reported detection of TMV in plants [33]. Also quite recently, a two-year average TMV detection rate of 55\% was reported [34], using 409 symptomatic tobacco leaf samples collected between 2010 and 2011 from Anhui, Fujian, Guangxi, Guizhou, Henan, Heliongjiang, Hubei, Hunan, Liaoning, Shandong, Shaanxi, and Yunnan. Consequently, early detection and effective control of TMV dispersal in China is crucial given that once a field is contaminated, decreasing and decontaminating TMV in soils and plant hosts is extremely difficult [14].

Using TMV coat-protein (CP) gene sequences isolated from China and available in the public database Genbank, this study employs a probabilistic Bayesian inferential phylodynamic framework to reconstruct the history of TMV's emergence and spatio-temporal diffusion throughout China's tobacco producing regions. In essence, this will entail; (i) estimation and inference of the evolutionary rate, time to emergence and geographical locations of key ancestral TMV 
variants, (ii) identification of statistically significant transmission pathways and quantifying levels of genetic diversity amongst TMV populations; (iii) describing the phylogenetic relationships amongst TMV sampled from different plant hosts throughout China.

\section{Materials and Methods}

\section{Viral Isolates}

A total of $110 \mathrm{TMV}$ coat-protein (CP) gene sequences retrieved from Genbank and isolated between the years 1997 and 2015 from 12 plant hosts that include; tobacco $(n=84)$, pepper $(n=10)$, eggplant $(\mathrm{n}=3)$, tomato $(\mathrm{n}=3)$, beans $(\mathrm{n}=3)$, pumpkin $(\mathrm{n}=1)$, Rehmannia glutinosa $(\mathrm{n}=1)$, Syringa oblate $(\mathrm{n}=1)$, Gynostemma pentaphyllum Makino $(\mathrm{n}=1)$, Atractylodes lancea $(\mathrm{n}=1)$, Agrimonia species $(\mathrm{n}=1)$ and Schisandra chinensis $(\mathrm{n}=1)$, displaying MD symptoms. These sequences were collected or sampled from 18 Chinese geographical locations encompassing 14 Chinese Provinces, two Central Government Controlled Municipalities and two Autonomous Regions. The corresponding respective sequence accession numbers, geographical sampling locations, sampling dates and plant host data used here are listed in Tables S1a and S1b in the Supplementary Information.

\section{Multiple sequence alignment and editing}

A multiple sequence alignment for the TMV CP gene dataset was prepared and aligned with MUSCLE [35], in MEGA v6.06 [36] and thereafter, manually edited using the Improved Alignment Editor (IMPALE: http://web.cbio.uct.ac.za/ arjun/) to maintain the readingframe integrity.

\section{Estimating levels of genetic diversity and variability}

The levels of genetic diversity in nucleotide sequence datasets for TMV CP were estimated by calculating the overall mean pairwise genetic distance (dissimilarity) first for the full dataset and secondly, for province-specific datasets in MEGA v6.06 [36] and the percentage sequence identity score (a measure of sequence similarity) using the Species Demarcation Tool v1.2 [37]. In addition, to reconstruct changes in TMV population size dynamics as a proxy for the estimate of the change in levels of genetic diversity amongst TMV pathogen populations circulating around China, the Bayesian SkyGrid nonparametric model coupled with a piece-wise constant function and a Gaussian Markov random field (GMRF) prior for temporal smoothing were used here.

\section{Detection and removal of evidence of molecular recombination}

The presence of recombinant sequence tracts was screened for using methods implemented in the Recombination Detection Program (RDP) [38].

\section{Best-fit nucleotide and evolutionary model selection}

The best-fit nucleotide substitution model for the TMV CP gene dataset was inferred using jModeltest v2.14 [39] whereas, the best-fit molecular clock and demographic models were determined using the marginal likelihood estimation methods of path sampling and stepping-stone [40], implemented in BEAST v1.8.1 [35], with the BEAGLE high performance library v2.1.2 [41].

\section{Phylogeographic inferential analyses}

Using the recombinant-free TMV-CP gene dataset, the most plausible evolutionary history and diffusion pathways utilized by TMV since its emergence in China, to achieve its current spatial distribution was reconstructed using the discretized phylogeographic model implemented in BEAST v1.8.1 [35,42]. Each of the five replicate BEAST runs conducted here had a discretized Monte Carlo Markov Chain (MCMC) of length ranging between $1.0 \times 10^{8}$ and $6.0 \times 10^{8}$. All BEAST run output $\log$ files where viewed in Tracer v1.6 [43], to confirm both good chain mixing and convergence of model parameters where the effective sample size (ESS) estimate values exceeded 200. LogCombiner v1.8.1 (BEAST embedded package) was then used to merge log and tree output files showing similar results from independent replicate BEAST runs. Next, Tree Annotator v1.8.1 (BEAST embedded package) was used to create an annotated maximum clade credibility (MCC) tree from the posterior distribution of trees produced in the MCMC analyses. The discretized TMV-CP MCC tree obtained here was visualized using FigTree v1.4.2 [44]. Thereafter, SpreaD3 v0.9.6 [45] was used to; (i) calculate Bayes factor statistical support for the most significant diffusion pathways used by TMV to disseminate around China and, (ii) create a karyotype markup language (KML) file containing the dispersal pathway history that can be visualized in Google Earth (https://earth.google.com/). Herein, TMV viral movements between sampling locations yielding a $\mathrm{BF}$ of $<5$ were taken as having negligible support; those with a BF equal to and/or $>5$ but $<10$, were viewed to have substantial support; and movements with $\mathrm{BF}$ values $>10$ but less than 100 had strong support, whereas those with $\mathrm{BF}$ values $>100$ were taken to have decisive statistical support respectively [46,47].

\section{Results and Discussion}

\section{Minimally variable mean pairwise genetic distance levels}

For the full TMV-CP dataset, an overall mean pairwise genetic distance (d) of 0.038 and $\geq 83 \%$ nucleotide sequence similarity were estimated using MEGA v.6.06 [36] and SDT [37] respectively. Whereas most sampling locations had $\mathrm{d}$ estimate values less than 0.015 , the highest overall mean pairwise genetic distances were observed for Henan $(\mathrm{d}=0.180)$; Hubei and Shandong $(\mathrm{d}=0.045)$; Yunnan $(\mathrm{d}=0.036)$; Sichuan $(\mathrm{d}=0.031)$; Chongqing and Guangxi $(\mathrm{d}=0.019)$, and Liaoning $(\mathrm{d}=0.015)$ respectively. Altogether, these estimates show that isolates used in this study were very closely related and are TMV.

\section{TMV has a small effective population size}

The effective population size (EPS) and not the census population size (CPS), matters more to the evolution of a virus population because it represents the number of individual viral particles within a given population that contribute equally to the following generation as opposed to just the number of particles within an infected plant host [48]. The CPS estimate is usually larger than that of the EPS because it accounts for mutants within the population that often cannot replicate and therefore play no part in increasing the EPS and/or adding to the genetic diversity. For this reason the EPS estimate is a better indicator of changes in viral population dynamics as compared to the CPS estimate. Given that the number and diversity of plant virus populations within a single infected host can be very large, where for example TMV's CPS in an infected tobacco leaf has been estimated to be in the range of 1011 to 1012 [49,50], estimation of the EPS range is 
important. Here, estimation of the changes in the EPS dynamics as a proxy for the estimated change in levels of genetic diversity amongst TMV populations using the Bayesian SkyGrid non-parametric demographic model prior (Figure 1) implemented in BEAST v.1.8.1 indicates that, TMV has a small EPS in the range of 101 to 104, which is inferred here as translating to low levels of genetic diversity. It is strongly believed that TMV's low intrinsic infectivity is one possible determinant of a small effective population size [51]. The EPS of TMV remained fairly constant at around 101 from 1924 until around 1997. After 1997, the EPS increased marginally above 102 up-to 2003, before falling in-between 2003 and 2007 to approximately 102, and increasing again between 2007 and 2011 to approximately 104, and finally decreasing to just under 102 from 2011 to 2015, the last sampling year. These four-year alternating cycles of low level EPS changes for TMV indicate that, the occurrence of genetic drift [52], is possibly high.

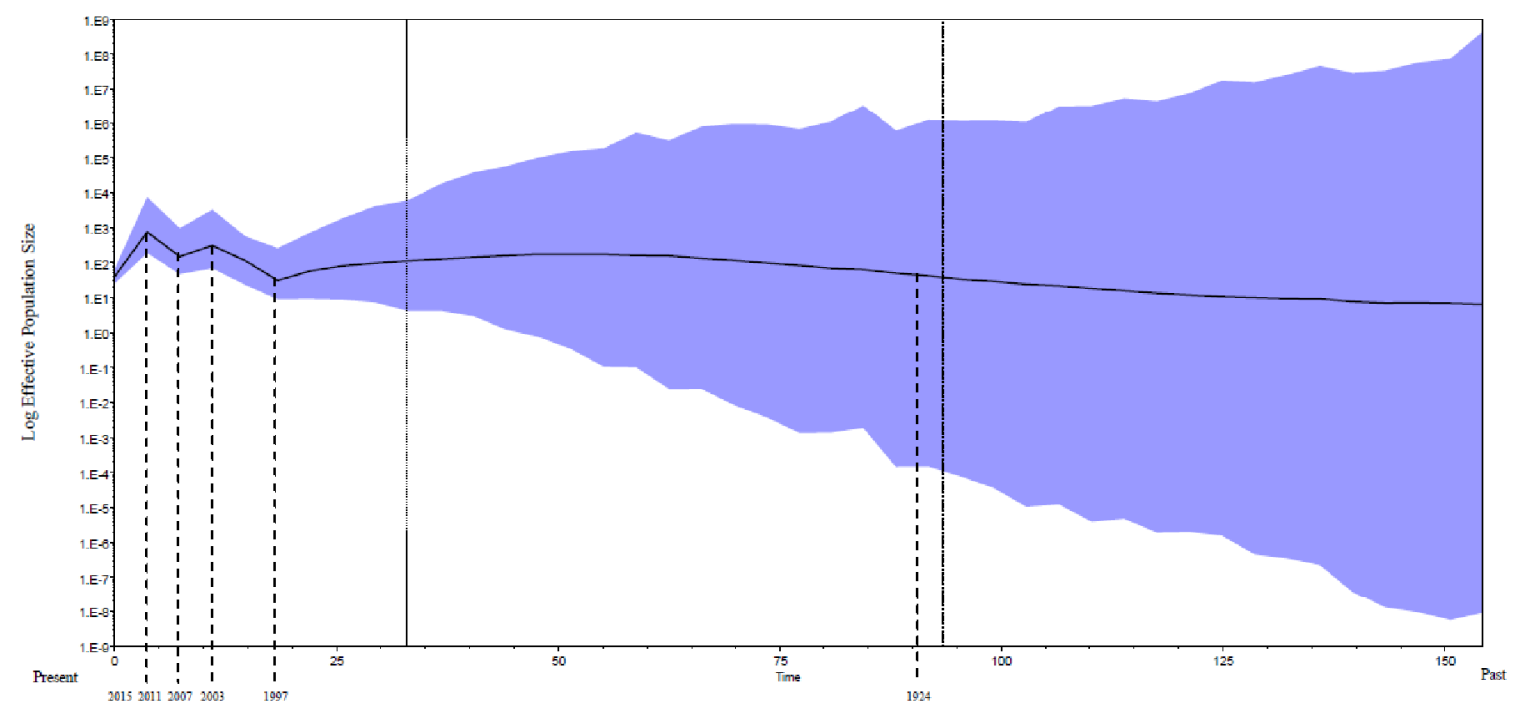

Figure 1: A Bayesian Skygrid demographic model plot displaying changes in TMV effective population sizes (EPS): TMV populations are shown here to have a small EPS in the range of 101 to 104, which is inferred here as translating to low levels of genetic diversity. The plot shows time from the past to the present in both "years before present" (YBP) and "calendar year" format, where "0" in the YBP format and "2015" in the calendar format each represent the most recent sampling date for the data. The horizontal solid black line represents the posterior median whereas the blue-shaded region represents the $95 \%$ Bayesian credibility interval of the effective population size Ne (t) based on the data. From right to left, the first dashed horizontal line represents the time-point for the inferred emergence of TMV in China's Henan province and the last four dashed vertical lines represent the time-points where significant $\mathrm{Ne}(\mathrm{t})$ changes in TMV populations occurred.

\section{Characterization of TMV recombinants}

No evidence for the presence of intra-strain recombinant sequence tracts was detected in the TMV-CP gene dataset using methods implemented in the RDP [38]. Consequently, the resulting recombinant-free TMV-CP gene dataset was imported into the phylogeographic inferential analyses framework implemented in BEAST v1.8.1 [35].

\section{Nucleotide substitution and evolutionary model choice}

The evolutionary and spatiotemporal diffusion history of TMV in China was inferred using the best-fit generalized time-reversible nucleotide substitution model with four gamma rate categories (GTR + G4) and the best-fit uncorrelated lognormal relaxed clock coupled with a constant size population demographic prior, under the discrete reversible phylogeographic diffusion model implementing the Bayesian stochastic search variable selection (BSSVS) procedure. Implementing the BSSVS enables the construction of a Bayes factor (BF) test that identifies significant non-zero migration (transition) rates and locations which are epidemiologically linked so as to provide the most parsimonious description of the phylogeographic diffusion process [42]. Marginal likelihoods obtained for both molecular clock and demographic models evaluated using path sampling and steppingstone methods [40], are displayed in Table S2 in the Supplementary Information.

\section{Where and when TMV first emerged in China}

Globally, the New World, centering about some part of Peru, Bolivia or Brazil has been implicated as being the original habitat of tobacco mosaic virus [53]. Here, using the discretized Bayesian phylogeographic diffusion inferential framework implemented in BEAST v1.8.1, the most recent common ancestor (MRCA) of TMV in China is inferred to have first emerged in Henan province (root posterior probability $=0.3027$ ) around 1924 (95\% HPD; 1860 to 1971) (Figure 2). Henan province is located in eastern central China, on the plain between the Yellow and Huaihe rivers. Strong evidence to corroborate this inference exists. For example, in 1912, Henan Public Agricultural Major School, now known as Henan Agricultural University, located in Zhengzhou, was established in Henan province and it advanced quickly through inter-collegial ties with universities in the United Kingdom, Japan and the United States where the tobacco industry was already very well established and wherefrom, traders were importing tobacco seed and cigarettes into China since the ratification of the Treaty of Tianjin [16]. Also, Chinese agronomic records indicate 
Page 5 of 9

that tobacco cultivation was introduced into Henan [54], and that Henan was the primary hub of China's tobacco production before being surpassed by Yunnan province in the late-1980s up until today $[1,18,32]$. This study infers that the expansion of the tobacco cultivation practice throughout and beyond Henan province heralded the genesis of the spatiotemporal diffusion of TMV's progenitor across China from province to municipality and/or autonomous region, until the pathogen achieved its current distribution range.

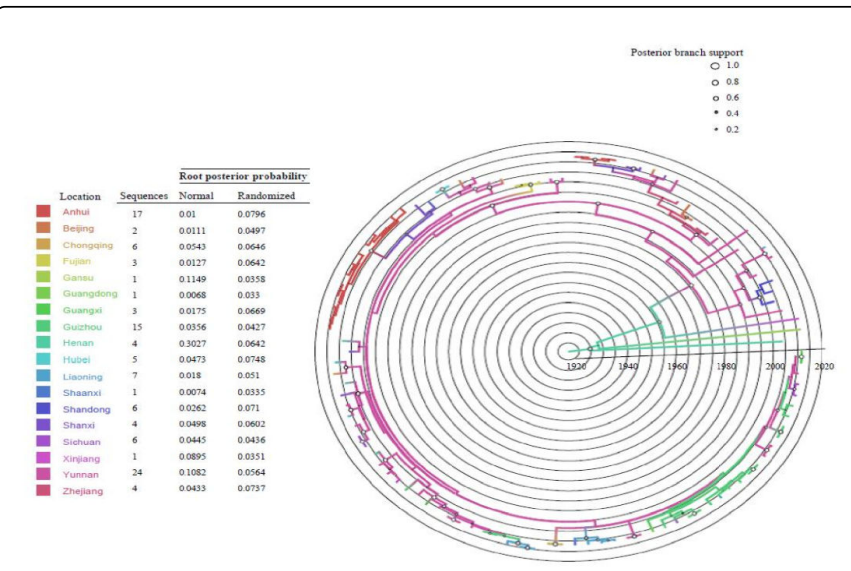

Figure 2: Temporally scaled maximum clade credibility (MCC) tree constructed using 110 recombination-free TMV coat protein (CP) sequences: Branches are colored relative to the 18 considered geographical locations from where the ancestral sequences represented by these branches most likely occurred. The posterior probability support of branches represented by circles at the tip nodes of branches, with circle sizes being proportional to the degree of branch support. Also indicated are the numbers of TMV-CP sample sequences used from each location considered, as well as the root posterior probabilities of each location being the origin of the most recent common ancestor of all sampled TMVs.

\section{The original and long-term plant host of TMV in China}

Several Nicotiana species commonly referred to as tobacco plants are cultivated as ornamental garden plants and those such as $N$. glutinosa and $N$. benthamiana, are model organisms for plant research $[53,55]$. Of these species, only the Nicotiana tabacum (N. tabacum) species is predominantly cultivated at commercial level worldwide for the production of tobacco leaf and other tobacco products such as cigarettes, cigars, and chewing tobacco [53], and the results obtained here indicate that $N$. tabacum, is unlikely to have been the first host of TMV in China. This is because land-use patterns were not as heavily devoted to tobacco cultivation prior to, and around 1915 and 1916, when tobacco cultivation began in the country in Henan, and Yuxi County, Yunnan [21,54]. However, N. tabacum became China's preferred long-term host following:

The expansion of the tobacco cultivation practice from traditional to non-traditional production regions.

The emergence of the TMV progenitor around 1924 in Henan and its spatiotemporal diffusion across proximal and distant provinces, municipalities and/or autonomous regions as a result of the latter. Results obtained here also indicate that, the 110 Chinese TMV sequences were collected from 12 species in 8 families (Solanaceae,
Legumineae, Cucurbitaceae, Asteraceae, Oleaceae, Rosaceae and Schisandraceae).

In view of the above, and because TMV sequences used here were collected from only 18 geographical locations distributed across China, results obtained here indicate that currently, TMV has a wide plant host distribution throughout China. Also, inclusion of novel viral sequences sampled from locations within China that were not considered here, should show that the distribution of the virus amongst plant hosts in China is much wider. Furthermore, the aforementioned TMV plant hosts, may be key reservoirs of the virus from which spillovers, and even perhaps host-switches, occur seasonally often during MD outbreaks, furthering the dispersal of the pathogen across China.

\section{The mean nucleotide substitution rate for TMV}

The estimated mean nucleotide substitution rate for the TMV-CP segment dataset was $1.09 \times 10^{-3}\left(95 \%\right.$ HPD: $6.59 \times 10^{-4}$ to $\left.1.58 \times 10^{-3}\right)$. While this estimate is about the average rate of nucleotide substitution for RNA viruses $[13,56]$, it is a marginally higher rate of nucleotide substitution as compared to that of $1.1 \times 10^{-5}$ (95\% HPD: $8.4 \times 10^{-7}$ to $2.5 \times 10^{-5}$ ) previously reported for the CP region of TMV [57] and it is significantly higher compared to that of $2.2 \times 10^{-8}$ reported for 320 tobamovirus CP-genes representing 25 species [58]. Given that the CPsegment of TMV is functionally constrained and reportedly under strong negative selection pressure [56], the mean nucleotide substitution rate reported here for TMV remains within the inferred and expected fivefold range around the mean rate for RNA viruses of around $\sim 10^{-3}[29]$.

\section{Major dissemination pathways used by TMV to achieve its current spatial and host distribution}

A total of 15 statistically well supported (i.e. with BF>5.0) epidemiological links and movement pathways were identified for the TMV-CP dataset using the discretized phylogeographic model for the 18 geographical locations considered (summarized in Figure 3 below and Table S3 in the Supplementary Information). Following its emergence in Henan province around 1924, TMV is inferred here to have disseminated to and localized its spread first within Yunnan between 1924 and 1976 and immediately thereafter again in Gansu between 1924 and 2015. This pair of transmission events was soon followed by a series of four separate TMV movements, three of these from Yunnan province: first to Zhejiang between 1993 and 1995, then to Shandong between 1994 and 1998, and to Fujian between 1995 and 1999; and the fourth in this series of successive movements was that from Zhejiang to Beijing between 1996 and 1998. From Yunnan, TMV also spread to Shanxi between 1999 and 2004; to Guizhou between 2002 and 2004; to Chongqing between 2002 and 2006; to Hubei between 2006 and 2011; to Sichuan between 2007 and 2011 and finally, to Guangxi between 2008 and 2012. Other TMV movements inferred here include that from Shandong to Anhui between 2003 and 2010; then from Liaoning to Shanxi between 2008 and 2011, and lastly, the only long distance transmission of the virus from Guizhou to Liaoning between 2009 and 2011. 


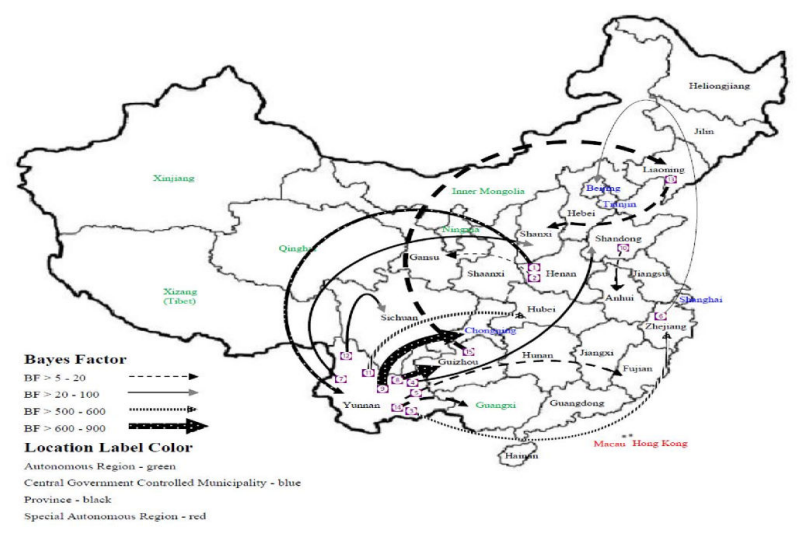

Figure 3: A summary of the 15 statistically supported epidemiological links and movement pathways between the 18 geographical locations considered here: Dashed and solid lines represent the degree of Bayes factor support and the thickness of the lines is proportional to the degree of Bayes factor support. Purple numbered white circles indicate the inferred sequential ordering of independent viral transmission/movement events amongst the locations considered.

\section{Yunnan is a source rather than a sink of TMV dispersal}

Yunnan province is China's leading tobacco production hub and annually it has well over 400, 000 hectares of land designated to tobacco cultivation [59]. Yunnan also accounts for $20 \%$ of the world's tobacco leaf yield; $40 \%$ of China's leaf yield and leaf exports respectively [60]. The Bayesian phylogeographic analyses of the TMV$\mathrm{CP}$ dataset inferred that, Yunnan province is apparently a major source of TMV and also a thoroughfare of trans-China TMV movements. The position that Yunnan is possibly a major source rather than a sink of TMV spatiotemporal diffusion across China is based on the fact that nine $(60 \%)$ of the 15 BF-supported TMV dissemination pathways inferred here were; (i) all outward-bound transmission events of the virus from Yunnan to other Chinese provinces, municipalities and/or autonomous regions and, (ii) the estimated timing of each of these dispersal events is congruent with detection of TMV in tobacco using electron microscopy in Yunnan [61] and detection and isolation of TMV from tobacco leaves collected in Yuxi, Qujing, Honghe, Dali, and Chuxiong counties, Yunnan province $[61,62]$. Together with Baoshan, Kunming, Wenshap, and Zhaoteng, the latter five Yunnan counties, are sub-suitable, suitable and most-suitable for tobacco cultivation and depending on this characterization, they each carry a measureable TMV threat to tobacco and other crops [60]. Results obtained here also indicate that, the outward-bound TMV transmissions from Yunnan may have occurred at a time when; (i) Yunnan had just usurped Henan as China's major tobacco production hub $[18,63]$, (ii) China extended the tobacco planting area from hub-provinces of Yunnan, Guizhou, Sichuan, Henan and municipalities such as Chongqing, into marginal lands and non-traditional production areas [33], (iii) Yunnan was listed together with Shaanxi, Shandong, Liaoning, Heliongjiang provinces, as a location wherein TMV susceptible, to moderately susceptible tobacco cultivars were widely grown prior to, and between 2007 and 2011 [64], and (iv) 95\% MD was reported in Yunnan [65]. These viral dispersal events partly explain the high, two-year average
TMV detection rate of more than 55\% reported throughout Anhui, Fujian, Guangxi, Guizhou, Henan, Heliongjiang, Hubei, Hunan, Liaoning, Shandong, Shaanxi, and Yunnan, between 2010 and 2011 respectively [34]. Invariably, the locations where TMV was detected in the above study contain marginal lands and non-traditional tobacco production areas.

The latter indicates that Yunnan, has significantly contributed far more than any other major tobacco production regions within China to the spatiotemporal diffusion of the virus throughout the country. In fact except for the single seeding of Yunnan by TMV from Henan, analyses conducted here did not reveal any other inward-bound transmissions of the virus into Yunnan. This may be because Yunnan is a donor rather than a recipient of TMV. Further inspection of outwardbound viral transmission movements from Yunnan indicates that there were far more introductions of virus into non-traditional and/or lowlevel tobacco production regions than there were movements of the virus into ready-established major tobacco production hubs such as Guizhou and Sichuan. The lower numbers of TMV movements reported here between Yunnan and other major production hubs may be the outcome of a strong protectionist policy that was exercised by premieres from the mid-1990's to the early 2000's to minimize the movement of and protect plants of economic importance from viral diseases $[20,66]$. Movements could also have been lower because the regions were more prepared to control the spread of the virus from the greenhouse to the field. In contrast, the scrapping of the inter-regional protectionist policy by the STMA and the CNTC possibly encouraged free movement of TMV-infected and/or highly susceptible plant materials between neighboring and/or proximal Chinese provinces, autonomous regions, and/or municipalities. Also, rapid conversion of neglected lands in non-traditional tobacco production regions, where lands where covered by weeds and/or other TMV-reservoirs, that could have increased the spread of the virus. Because, non-traditional tobacco production regions were probably not equipped to manage TMV, its spread into and within such areas would have been relatively easier and quicker. Such a scenario, would explain why more TMV movements from Yunnan to minority production areas were inferred here.

\section{Possibly significant determinants of TMV spatiotemporal diffusion}

Several factors appear to have aided the spatiotemporal diffusion of TMV across China since its inferred emergence in Henan. Chief amongst these is: (i) the limited sources of natural genetic resistance to TMV [64]; (ii) over-reliance on both $\mathrm{N}$ and $\mathrm{N}^{\prime}$ gene mediated resistance in tobacco which is easily overcome by several mutant strains of the virus such as TMV-O [33,67-69]; (iii) reportedly high TMV detection rates ranging between $48 \%$ to $100 \%$ and MD incidences greater than $95 \%$ occurring within tobacco seedlings before and after transplanting from float tray production systems [65]. Another significant determinant is an increasing population of new tobacco farmers either lacking or receiving limited training in control and management of the virus from float trays, within greenhouses, and under field conditions. This leads to increased cultivation of TMV susceptible or moderately susceptible tobacco cultivars and increased losses due to the virus $[33,34,64]$. The dispersal of TMV throughout China is especially aided by the limited introduction, availability, affordability, distribution and breeding of resistant or moderately resistant cultivars (e.g. Coker86, Coker176, CV87, Zhongyan90 and Jiyan5) [64]. 


\section{Controlling TMV dispersal throughout China}

Controlling TMV dispersal throughout China is increasingly challenging because major tobacco production hubs such as Yunnan, Guizhou, Henan, and Sichuan, experience high TMV detection rates and high MD incidences [34]. Just as these regions seed and spread TMV within and between themselves, they also introduce the virus into new and/or non-traditional tobacco production areas. Therefore, successful control and management of TMV diffusion throughout China hinges on meticulous, consistent, stringent implementation of an integrated approach that stipulates good agronomic practices and farmer training in hub regions, traditional and non-traditional tobacco production areas. In response to the increasing threat posed by TMV on tobacco and other plant species, Chinese researchers have put forward several strategies to help control the spread of virus. The first and most significant of these responses was the development of TMV resistant transgenic tobacco as early as 1992 [56]. Against strong opposition from both local and foreign farmers, tobacco and cigarette manufacturing companies, around 1994, cultivation of transgenic TMV resistant tobacco was revoked and remains to this day prohibited within China [56,70]. While the prohibition on cultivation of transgenic TMV resistant tobacco persists and is a huge setback on efforts to control the spread of the virus, China is at the forefront in developing molecular diagnostic technologies for rapid, robust and specific detection of TMV within soils and plant hosts. As an example, a One-step Immunocapture Real Time Polymerase Chain Reaction (RT-PCR) assay to detect the presence and quantify the load of the virus in soils was recently developed [71]. Additionally, a multiplex PCR assay for rapid detection of TMV and other tobamoviruses sampled from soil and plant hosts throughout China has since been developed [72], and a novel approach to discriminate TMV-infected tobacco based on laser-induced spectroscopy (LIBS) has been proposed [73]. It is clear then that, molecular diagnostic technologies for TMV such as those highlighted above, are good indicators of healthy and/or contaminated soils and/or plant hosts. These technologies permit early farmer intervention by way of decontamination and decreasing levels of the virus in soils, plants, and/or tools through sterilization, or use of biological, chemical and/or many other integrated MD management approaches.

The use of biocontrol agents such as, Pseudomonas putida A3-m strain [14], a pathogen isolated from tobacco rhizosphere soil and whose anti-TMV activity has been studied in vivo [74], has demonstrated the inactivation of TMV in field agricultural soil, helping to minimize early crop infections. Similarly, investigations for high efficacy, TMV biological and/or chemical pesticide controls have led to increased identification, availability and understanding of the mechanistic action underlying the functionality of efficient anti-TMV compounds. Compounds shown to exhibit a high efficacy against TMV include benzolactones [75], benzofuran derivatives [76], biphenyls from Garnicia tetralata [77], Ningnanmycin (NNM), Antofine (ATF), Dufulin (DFL), Bingqingxiao (BQX) [78], and Alpha-momorcharin [79]. Most of these anti-TMV biocontrol agents activate the systemic acquired resistance (SAR) response, thereby affording the plant protection against invading virus $[67,80]$. Only recently Chinese researchers revealed that the plant immune response against TMV in tobacco strengthens due to the presence of residues of the herbicide Bensulfuron-methyl (BSM) in treated tobacco plants and soils. The latter then results in increased levels of the phytohormones, jasmonic acid (JA) and salicylic acid (SA); up-regulation of NtRDR1 and NtRDR6 genes key to fighting TMV and the expression of target genes (NtWIPK, NtPRla, NtPAL) in the JA and SA signaling pathway [81].
In fact it has recently been reported that salicyclic acid and jasmonic acid are both essential for systemic resistance against TMV in tobacco (Nicotiana benthamiana) [55]. Similarly, a coat protein mediatedresistance (CP-MR) strategy against TMV that is not affected by either the form or the inoculum concentration was established in China for Rehmannia glutinosa, a comprehensive traditional Chinese medicinal herb. $R$. glutinosa is one of the many TMV susceptible or moderately susceptible crops rotated with tobacco under field conditions in major tobacco production areas throughout provinces, municipalities, and/or autonomous regions in China, and consequently, its cultivation suffers from infection by TMV [82]. Therefore, controlling the spread of TMV in plant hosts such as R. glutinosa, is a major step in the fight against MD.

\section{Sources of resistance to TMV infection}

Globally, the best resistance source to TMV infection is the $N$ gene from Nicotiana glutinosa tobacco cultivars. Infection by TMV of Nicotiana species native to Central and South America: N. glutinosa is a native of Peru, N. repanda of Mexico, N. rustica of Ecuador and Peru, and $N$. langsdorfii of Brazil, elicits a hypersensitive response. In contrast, Nicotiana species from North America, southern South America, and Australia are most susceptible to TMV infection and have high virus titres [53]. The results obtained here suggest that seed for TMV susceptible Nicotiana species was most probably introduced into China from North America and Europe through the Treaty of Tianjin. This probably explains why $N$ gene carrying tobacco cultivars are very few in China, because they are not indigenous to the country. Because TMV resistance sources are limited in China, this leads to high yield losses due MD [64]. While use of cultivars harboring the $N$ gene would reduce yield losses and MD outbreaks, it is important to clarify that resistance to TMV in N gene carrying tobacco cultivars is not absolute. This is because, (i) $N$ gene mediated resistance is suppressed at temperatures of $28^{\circ} \mathrm{C}$ and above, allowing TMV to spread [69] and, (ii) the $N$ gene is a member of the TIR-NBS-LRR class of plant resistance genes and so deletion and/or point mutations of TIR can interfere with the wild-type $N$ gene function, increasing the susceptibility of cultivars to TMV infection [83]. While it is the most effective resistance source of controlling TMV, introgression of the $N$ gene into tobacco cultivars is associated with reduced yield and reduced yield quality [67], particularly so into $N$. tabacum, a variety popular globally and with Chinese farmers in major production areas $[33,64]$. So, to increase the yield and quality of tobacco, farmers in China must make use of an integrated disease management system. This system must stipulate breeding for and developing novel resistance sources, and environmentally friendly anti-TMV biocontrol agents. It should also encourage early and regular detection of TMV from soils and plant tissue using molecular diagnostic tools, decontamination and decreasing of pathogen levels in soils and plant hosts, and good agronomic practices such as crop rotation and use of sterile seed and farming equipment. If an integrated approach is not implemented, controlling the spread of TMV within and beyond China's major tobacco production regions will remain a challenge. Farmers, tobacco companies and industry stakeholders will also continue to face the threat of dwindling revenues and increasing costs of MD management.

\section{Conclusion}

TMV is inferred here to have emerged in China in Henan province around 1924. Although its mean substitution rate is marginally higher 
than previously reported rates, the rate estimated here for the virus remains within the inferred mean evolutionary range for RNA plant viruses. The virus has achieved its current wide spatial and plant host distribution across China through, (i) 15 BF-supported diffusion pathways, $60 \%$ of which are outward bound transmission events from Yunnan province to other sampling locations and, (ii) a growing shift toward cost-efficient tobacco crop substitution alternatives and adoption of a mixed-crop farming system throughout China. The Bayesian phylogeographic analyses conducted here also suggest that Yunnan province is most probably a source, rather than a sink of TMV dispersal throughout China, and a major thoroughfare of trans-China TMV movements. Finally, results obtained here indicate that TMV populations exhibited both low effective population sizes and levels of genetic diversity, while individuals from distinct hosts were phylogenetically similar probably due to strong bottlenecks and purifying selection.

\section{References}

1. Akinyemi IA, Wang F, Zhou B, Qi S, Wu Q, et al. (2016) Ecogenomic survey of plant viruses infecting tobacco by next generation sequencing. Virol J 13: 181.

2. FAO (2003) Issues in the global tobacco economy 2-4.

3. Li C (2012) The political mapping of china's tobacco industry and antismoking campaign. John L. Thornton China Center Monograph Series, Washington DC: Brookings.

4. Lartey RT, Voss TC, Melcher U (1996) Tobamovirus evolution: Gene overlaps, recombination and taxonomic implications. Mol Biol Evol 13: 1327-1338.

5. Chase AR (2014) The truth about milk and TMV. Dis Manag 14-16.

6. Spence NJ, Sealy I, Mills PR, Foster GD (2001) Characterisation of a tobamovirus from trailing petunias. Eur J Plant Pathol 107: 633-638.

7. Alexandre MAV, Soares RM, Rivas EB, Duarte LML, Chagas CM, et al. (2000) Characterization of a strain of tobacco mosaic virus from petunia. J Phytopathol 148: 601-607.

8. Arli-Sokmen M, Mennan H, Sevik MA, Ecevit O (2005) Occurrence of viruses in field-grown pepper crops and some of their reservoir weed hosts in samsun, turkey. Phytoparasitica 33: 347-358.

9. Choi SK, Yoon JY, Chung BN (2009) Genome analysis and characterization of a tobacco mosaic virus isolate infecting balsam (Impatiens balsamina). Arch Virol 154: 881-885.

10. Kumar S, Udaya Shankar AC, Nayaka SC, Lund OS, Prakash HS, et al. (2011) Detection of tobacco mosaic virus and tomato mosaic virus in pepper and tomato by multiplex RT-PCR. Lett Appl Microbiol 53: 359-363.

11. Nassar EA, El-Dougdoug KA, Osman ME, Dawoud RA, Kinawy AH, et al. (2012) Characterization and elimination of a TMV isolate infecting chrysanthemum plants in Egypt. Int J Virol 8: 14-26.

12. Broadbent L (1965) The epidemiology of tomato mosaic. Seedtransmission of TMV. Ann Appl Biol 56: 177-205.

13. Hollings $\mathrm{M}$, Huttinga $\mathrm{H}$ (1976) Tomato mosaic virus. CMI/AAB descriptions of plant viruses, no. 156. Commonwealth Mycology Institute/Association for Applied Biology, Kew.

14. Yang J, Guo C, Zhai X, Shen L, Qian Y, et al. (2012) Inactivation of tobacco mosaic virus in soil by pseudomonas putida A3-m strain to prevent virus mosaic disease. Afr. J. Microbiol. Res 6: 6300-6307.

15. Bennedict C (2011) Golden-silk smoke: A history of tobacco in china. Berkeley: University of California Press.

16. Cavendish R (2008) The treaties of Tianjin. History Today 58.

17. Mackay J, Eriksen M (2002) The tobacco atlas, (2nd edition), Geneva: World Health Organization 18-19.

18. Peng Y (1997) Smoke and Power: The political economy of chinese tobacco. University of Oregon, PhD thesis. In: Beyer de J, Kollars N,
Edwards N, Cheung H (2004) Health, Nutrition and Population (HNP) Discussion Paper. Economics of Tobacco Control 21. Research on Tobacco in China: An annotated bibliography of research on tobacco use, health effects, policies, farming and industry. The International Bank for Reconstruction and Development/The World Bank 4-12.

19. Cochran S (1980) Big Business in China: Sino-Foreign rivalry in the cigarette industry, 1890 - 1930. Harvard Studies in Business History 33.

20. Fang J, Lee K, Nidhi S (2017) The china national tobacco corporation: From domestic to global dragon?. Glob Public Health 12: 315-334.

21. Maeda Y, Yongqing H, Yunling Z (2003) A study of the tobacco sector in selected provinces in cambodia and china. Working Paper 185: Sectoral Activities Programme, International Labour Office 31-35.

22. Bhatia SL (2012) Exploring China's formidable cigarette industry.

23. http://www.chinalawedu.com/falvfagui/fg22016/12453.shtml

24. http://www.chinalawedu.com/falvfagui/fg22016/12441.shtml

25. Campaign for tobacco-free kids (2012) The Chinese Tobacco Market and Industry Profile 1-24.

26. Hu TW (2008) Tobacco control policy analysis in china: Economics and Health. London: World Scientific Publishing Company 189-210.

27. Todd A (2007) Turning Over a New Leaf. USDA rural development: Report on tobacco production in China.

28. He P, Takeuchi T, Yano E (2013) An overview of the china national tobacco corporation and state tobacco monopoly administration. Environ. Health Prev. Med 18: 85-90.

29. Holmes EC (2003) Molecular clocks and the puzzle of RNA virus origins. J. Virol 77: 3893-3897.

30. State Council (2006) People's Republic of China Temporary Law on Tobacco Leaf Tax.

31. Hu T, Mao Z, Shi J, Chen W (2010) The role of taxation in tobacco control and its potential economic impact in china. Tob. Control 19: 58-64.

32. Li VC, Wang Q, Xia N, Tang S, Wang CC, et al. (2012) Tobacco crop substitution: Pilot effort in china. Am. J. Public Health 102: 1660-1663.

33. Ruitai C, Xianchao Z, Zhifa W, Zhenye G, Hansong D, et al. (1997) A report investigating and studying tobacco infectious diseases of 16 main tobacco production provinces (regions) in China. Chinese Tob. Sci 4: 1-7.

34. Wu K, Chen W, Luo Z, Wang B, Cheng J, et al. (2016) Distribution and molecular variability of four tobacco viruses in China. Virologica Sinica 31: 525-527.

35. Drummond AJ, Rambaut A (2007) BEAST: Bayesian evolutionary analysis by sampling trees. BMC Evol Biol 7: 214 .

36. Tamura K, Peterson D, Peterson N, Stecher G, Nei M, et al. (2013) MEGA version 6.06: molecular evolutionary genetic analyses using maximum likelihood, evolutionary distance, and maximum parsimony methods. Mol Biol Evol 28: 2731-2739.

37. Muhire BM, Varsani A, Martin DP (2014) SDT: A virus classification tool based on pairwise sequence alignment and identity calculation. PLoS One 9: e108277.

38. Martin DP, Murrell B, Golden M, Khoosal A, Muhire B, et al. (2015) RDP4: Detection and analyses of recombination patterns in virus genomes. Virus Evol 1: 1-5.

39. Darriba D, Taboada GL, Doallo R, Posada D (2012) jModeltest2: more models, new heuristics and parallel computing. Nature Med 9: 772.

40. Baele G, Lemey P, Bedford T, Rambaut A, Suchard MA, et al. (2012) Improving the accuracy of demographic and molecular clock model comparison while accommodating phylogenetic uncertainty. Mol Biol Evol 30: 2157-2167.

41. Ayres D, Darling A, Zwickl D, Beerli P, Holder M, et al. (2011) BEAGLE: an application programming interface and high-performance computing library for statistical phylogenetics. Syst Biol 61: 170-173.

42. Lemey P, Rambaut A, Drummond AJ, Suchard AM (2009) Bayesian phylogeography finds its roots. PLOS Comput. Biol 5: e1000520.

43. http://tree.bio.ed.ac.uk/software/tracer/

44. http://tree.bio.ed.ac.uk/software/figtree/ 
Page 9 of 9

45. Bielejec F, Baele G, Vracken B, Suchard MA, Rambaut A, et al. (2016) SpreaD3. interactive visualization of spatiotemporal history and trait evolutionary processes. Mol Biol Evol 33: 2167-2169.

46. Kass RE, Raftery AE (1995) Bayes factors. J Am Stat Assoc 90: 773-795.

47. Suchard MA, Weiss RE, Sinsheimer JS (2001) Bayesian selection of continuous-time Markov chain evolutionary models. Mol Biol Evol 18: 1001-1013.

48. Gill MS, Lemey P, Faria NR, Rambaut A, Shapiro B, et al. (2013) Improving Bayesian population dynamics inference: a coalescent-based model for multiple loci. Mol Biol Evol 30: 713-724.

49. Harrison BD (1956) The infectivity of extracts made from leaves at intervals after inoculation with viruses. J Gen Microbiol 15: 210-220.

50. Malpica JM, Fraile A, Moreno I, Obies CI, Drake JW, et al. (2002) The rate and character of spontaneous mutation in an RNA virus. Genetics 162: $1505-1511$.

51. Garcia-Arenal F, Palukaitis P, Zaitlin M (1984) Strains and mutants of tobacco mosaic virus are both found in virus derived from single- 24 . lesion-passaged innoculum. Virology 132: 131-137.

52. Sacristan S, Malpica J M, Fraile A, Garcia-Arenal F (2003) Estimation of population bottlenecks during systemic movement of tobacco mosaic virus in tobacco plants. J Virol 77: 9906-9911.

53. Holmes EC (1951) Indications of a new world origin of tobacco mosaic virus. Phytopathology 41: 341-349.

54. https://www.britannica.com/place/Henan/Economy

55. Zhu F, Xi DH, Yuan S, Xu F, Zhang DW, et al. (2014) Salicyclic acid and jasmonic acid are essential for systemic resistance against Tobacco mosaic virus in Nicotiana benthamiana. Mol Plant Microbe Interact 27: 567-577.

56. Jia S, Peng Y (2002) GMO biosafety research in china. Environ Biosafety Res 1: 5-8.

57. Jenkins GM, Rambaut A, Pybus OG, Holmes EC (2002) Rates of molecular evolution in RNA viruses: A quantitative phylogenetic analyses. J Mol Evol 54: 154-165.

58. Gülser C, YIlmaz NK, Candemir F (2008) Accumulation of Tobacco mosaic virus (TMV) at different depths clay and loamy sand textural soils due to tobacco waste application. Environ. Monit. Assess 146: 235-242.

59. Li C, He X, Zhu S, Zhou H, Wang Y, et al. (2009) Crop diversity for yield increase. PLoS One 4: e8049.

60. Zhang L, Yu Y, Lin Z, Huang Z (2016) Strengthening science transmission capability: A case of Yunnan experience in disseminating the technology to farmers and field technicians in the tobacco production chain. Yuxi Branch of Yunnan Tobacco Company of CNTC, China.

61. Zhang ZK, Li YG, Fang Q, Mei WQ, Li YH, et al. (1992) Using technique of electron microscopy detecting the pathogens of Yunnan tobacco virus diseases. Journal of South China Agricultural University 27-29.

62. Ming D, Qi F, Lizhen Z, Luming Y, Jiling W, et al. (2004) Comparison of the coat protein gene sequence of tobacco mosaic virus isolated from different regions in Yunnan province. Virol Sin 19: 76-78.

63. Zhifu W (1993) Henan tries to regain the crown. Tobacco International 195: 40 - 41.

64. Chen W, Huang T, Dai J, Liu W, Cheng J, et al. (2014) Evaluations of tobacco cultivars resistance to tobacco mosaic virus and Potato virus Y. Plant Pathol J 13: 37-43.

65. Yong L, Ying-Lin L (2006) Detection of major viral diseases in tobacco floating seedbed system in Yunnan province. Tobacco Science \& Technology 11: 58-61.
66. Zhou H (2000) Fiscal decentralization and the development of the tobacco industry in China. China Economic Review 11: 114-133.

67. Bagley CA (2001) Controlling tobacco mosaic virus in tobacco through resistance. Virginia Polytechnic Institute and State University, Virginia, USA.

68. Csillery G, Tobias I, Rusko J (1983) A new pepper strain of tomato mosaic virus. Acta Phytopathologica Academiae Scientiarum Hungaricae 18: $195-200$

69. Whitham S, McCormick S, Baker B (1996) The N gene of tobacco confers resistance to tobacco mosaic virus in transgenic tomato. Proceeding of the National Academy of Sciences 93: 8776-8781.

70. Wong AYT, Chan AWK (2016) Genetically modified foods in China and the United States: A primer of regulation and intellectual property protection. Food Science and Human Wellness 5: 124-140.

71. Yang JG, Wang FL, Chen DX, Shen LL, Qian YM, et al. (2012) Development of a one-step immunocapture real-time RT-PCR assay for detection of Tobacco Mosaic Virus in soil. Sensors 12: 16685-16694.

72. Dai J, Cheng J, Huang T, Zheng X, Wu Y, et al. (2012) A multiplex reverse transcription PCR assay for simultaneous detection of five tobacco viruses in tobacco plants. J Virol Methods 183: 57-62.

73. Peng J, Song K, Zhu H, Kong W, Liu F, et al. (2017) Fast detection of tobacco mosaic virus infected tobacco using laser-induced breakdown spectroscopy. Sci Rep 16: 44551.

74. Guo C, Yang JG, Shen LL, Qiang YM, Huang J, et al. (2011) The screening and identification of a Pseudomonas putid a against TMV. J. South China Normal Univ 32: 57-60.

75. Shen Q, Xu X, Zhang F, Xiang N, He P, et al. (2015) Two new benzolactones from the leaves of Nicotiana tabacum and their antitobacco mosaic virus activities. Nat. Prod. Res 30: 1464-1469.

76. Liu C, Shen Q, Zhang F, Zhang T, He P, et al. (2015) A new benzofuran derivatives from flue-cured tobacco and its anti-tobacco mosaic virus activity. Asian J. Chem 27: 2753-2755.

77. Hu QF, Wang YD, Zhu DL, Yu ZH, Zhan JB, et al. (2016) Three new biphenyls from the twigs of Garnicia tetralata and their anti-tobacco mosaic virus activity. J Asian Nat Prod Res 18: 1115-1121.

78. Li X, Chen Z, Jin L, Hu D, Yang S, et al. (2016) New strategies and methods to study interactions between tobacco mosaic virus coat protein and its inhibitors. Int J Mol Sci 17: 252.

79. Yang T, Zhu L, Meng Y, Lv R, Zhou Z, et al. (2017) Alpha-momorcharin enhances Tobacco mosaic virus resistance in tobaccoNN by manipulating jasmonic acid-salicylic acid crosstalk. J. Plant Physiol.

80. Shulaev V, Leon J, Raskin I (1995) Is salicyclic acid a translocated signal of systemic acquired resistance in tobacco? Plant Cell 7: 1691-1701.

81. Li R, Islam SU, Wu Z, Ye X (2016) Bensulfuron-Methyl treatment of soil affects the infestation of whitefly, aphid, and tobacco mosaic virus on Nicotiana tabacum. Front Plant Sci 7: 1970.

82. Teng Z, Shen Y, Li J, Lin Z, Chen M, et al. (2016) Construction and quality analysis of transgenic Rehmannia glutinosa containing tmv and cmv coat protein. Molecules 21: 1134.

83. Takabatake R, Seo S, Mitsuhara I, Tsuda S, Ohashi Y, et al. (2006) Accumulation of the two transcripts of the $\mathrm{N}$ gene, conferring resistance to tobacco mosaic virus, is probably important for $\mathrm{N}$ gene-development hypersensitive cell death. Plant Cell Physiol 47: 254-261. 